\title{
Generation of Bright, Tunable, Polarized $\gamma$-Ray Sources by Scattering Laser Pulses from APS Electron Beams
}

\author{
Y. Li, Y. Chae, L. Emery, Z. Huang, K. Harkay, J. Lewellen, S. V. Milton, and V. Sajaev \\ Advanced Photon Source, Argonne National Laboratory, Argonne, IL 60439
}

August 1, 2003

\begin{abstract}
We calculate the performance of possible Advanced Photon Source (APS) $\gamma$-ray sources for applications in nuclear physics research. For the APS storage ring, it is possible to generate tagged $\gamma$-ray photon fluxes of $10^{8}$, $0.7 \times 10^{8}$, and $0.3 \times 10^{8}$ photons/s at photon energies of $1,1.7$, and $2.8 \mathrm{GeV}$, respectively. For untagged photons, fluxes higher than $10^{8}$ photons/s are possible for those energies. For the injection booster, an untagged $\gamma$-ray photon flux up to $10^{8}$ photons/s at energy ranging from $5 \mathrm{MeV}$ to $1 \mathrm{GeV}$ is possible. This can be achieved using off-the-shelf commercial Ti:Sa laser systems. The photon fluxes predicted here are in general one to two orders of magnitude higher than facilities with similar photon energies.
\end{abstract}

\section{Introduction}

The use of Compton scattering of laser beams from high-energy electron beams to generate high-energy photons was proposed in 1963 [1,2] and has since been implemented using commercial lasers on electron storage rings to produce highly polarized photons in the energy range useful for nuclear physics [3-7].

Most of the existing facilities are built at synchrotron light sources such as GRAAL at ESRF [4], LEPS at SPring-8 [5], and LEGS at NSLS [6], where the stored beam is used as the electron source. As such, the depletion of the stored electron beam is the main obstacle to generating higher photon fluxes. As a result, the photon flux obtained is normally limited at a level of about $10^{6} / \mathrm{s}$ [3]. Only the future high intensity gamma-ray source (HIGS), based on the intracavity scattering with a free-electron laser at Duke University, boasts a photon flux higher than $10^{7} / \mathrm{s}$ while the highest photon energy is limited at $200 \mathrm{MeV}$ [7].

In this note, we propose to use the stored electron beams in the APS storage ring or the injection booster for generating $\gamma$-rays where the effect of the electron depletion can be mitigated or is nonexistent. For the storage ring, since it is operating in the "top-up" mode, i.e., the stored electron beam is replenished by frequent injections, the depletion of the beam can be easily compensated by either higher injection charge or more frequent injections. The relatively infrequent injection, on the other hand, makes it possible to store beams in the injection booster solely for generating Compton $\gamma$-rays between injections. Calculations show that the photon fluxes (both tagged and untagged) that can be generated is one to two orders of magnitude higher that those of the existing facilities in the same photon energy range.

\section{APS Compton scattering source performance}

\subsection{APS storage ring and the injection booster}

The APS comprises four successive accelerators: the injection linac, the particle accumulator ring (PAR), the injection booster, and the APS storage ring. In this note, we consider the booster and the storage ring as the potential electron beam sources. The beam parameters of these two rings are listed in Table 1.

The role of the injection booster is to ramp up the energy of the electron bunches from the injection linac or PAR from $400 \mathrm{MeV}$ to $7 \mathrm{GeV}$, and inject the bunches into the APS storage ring, where the beam is stored to 
Table 1. APS Booster and Storage Ring (SR) Beam Parameters

\begin{tabular}{|c|c|c|c|}
\hline & Booster & $\begin{array}{c}\text { APS SR } \\
\text { ID }\end{array}$ & $\begin{array}{c}\text { APS SR } \\
\text { BM }\end{array}$ \\
\hline Revolution frequency $(\mathrm{kHz})$ & 815 & \multicolumn{2}{|c|}{272} \\
\hline Injection frequency $F_{\mathrm{i}}(\mathrm{Hz})$ & 2 & \multicolumn{2}{|c|}{0.008} \\
\hline Radio frequency (MHz) & 352 & \multicolumn{2}{|c|}{352} \\
\hline Harmonic number & 432 & \multicolumn{2}{|c|}{1296} \\
\hline Nominal energy $(\mathrm{GeV})$ & 7 & \multicolumn{2}{|c|}{7} \\
\hline Stored beam energy $(\mathrm{GeV})$ & $0.45-4$ & \multicolumn{2}{|c|}{7} \\
\hline Energy gain per turn $(\mathrm{keV})$ & 32.0 & \multicolumn{2}{|c|}{-} \\
\hline Energy spread, rms@7-GeV & $0.1 \%$ & \multicolumn{2}{|c|}{$0.1 \%$} \\
\hline Emittance $\varepsilon_{0} @ 7 \mathrm{GeV}$ (m-rad) & $130 \times 10^{-9} a$ & \multicolumn{2}{|c|}{$2.5 \times 10^{-9}$} \\
\hline Coupling factor $k$ & $0.1^{b}$ & \multicolumn{2}{|c|}{$0.01-0.03$} \\
\hline Electrons per bunch & $6.25 \times 10^{10}(10 \mathrm{nC})^{c}$ & \multicolumn{2}{|c|}{$10^{11}(15 \mathrm{nC})$} \\
\hline Number of bunches & 1 & \multicolumn{2}{|c|}{24} \\
\hline Bunch repetition rate $(\mathrm{kHz})$ & 815 & \multicolumn{2}{|c|}{6528} \\
\hline Bunch length, rms,@7 GeV (ps) & 77 & \multicolumn{2}{|c|}{45} \\
\hline Beta functions $\beta_{\mathrm{x}, \mathrm{y}}(\mathrm{m})$ & $16,2.7$ & $19.5,2.9$ & $2.12,26.1$ \\
\hline Beam size $\sigma_{\mathrm{x}}, \sigma_{\mathrm{y}}(\mu \mathrm{m})^{d}$ & 786,102 & $274,8.5$ & $91.8,25.5$ \\
\hline Beam divergence $\sigma_{\mathrm{x}}, \sigma_{\mathrm{y}}(\mu \mathrm{rad})^{d}$ & 90,219 & $11.3,2.9$ & $56.3,1.1$ \\
\hline
\end{tabular}

a. Recent enhancement to the focus of the magnets has improved this down to $93 \mathrm{~nm}$ rad [8].

b. The booster may have a much smaller $\mathrm{k}$, but is not routinely measured.

c. This is determined by the radiation safety envelope. The highest ever achieved is $4-5 \mathrm{nC}$.

d. These are with $\mathrm{k}=1 \%$

generate synchrotron radiation for users. The booster is capable of storing electron beams with energy up to $4 \mathrm{GeV}$ and a duty cycle of $2 \mathrm{~Hz}$. The beam rate, determined by the circumference of the ring, is $815 \mathrm{kHz}$. The APS storage ring (SR), with a circumference three times that of the booster, stores electrons at up to $7 \mathrm{GeV}$ of energy, normally with 24 bunches, each with $15 \mathrm{nC}$ of charge.

Unique to the operation of the APS is the "top-up" operation mode, in which frequent injections are made into the storage ring to compensate for the lost electrons. Currently, injection is at 2-3 $\mathrm{nC}$ every two mins, indicating a beam decay rate of $10^{8} \mathrm{e}^{-}$per second. Note that, in the current transverse injection scheme, a beam perturbation is induced at every injection by displacement of the beam during the injection. The 2-min injection interval is determined by the balance between maximum tolerable beam perturbation by the users and a beam current stability of about $1 \%$.

A perturbation-minimized longitudinal injection scheme has been demonstrated. This new scheme enables more frequent injections but will need a different lattice for operation [9]. If implemented, injection at the booster duty cycle of up to $2 \mathrm{~Hz}$ is possible.

\subsection{Possible performance of the proposed $\gamma$-ray sources}

The maximum photon energy and the corresponding scattering cross section as a function of the electron beam energy accessible at the APS are plotted in Figs. 1 (a) and (b) for laser photon energies of 1.55, 3.10, and $6.20 \mathrm{eV}$. The laser is a Ti:Sa laser system working at the fundamental, second, and fourth harmonics (see Section 2.4 for laser requirement). Also plotted in Fig. 1 (c) is the maximum transferable linear polarization. The calculations are based on the formula given in Appendix A. With the booster, photon energies from a few $\mathrm{MeV}$ to $1 \mathrm{GeV}$ are possible, while with the SR the highest photon energy accessible is $2.8 \mathrm{GeV}$ at $7 \mathrm{GeV}$ electron beam energy. 

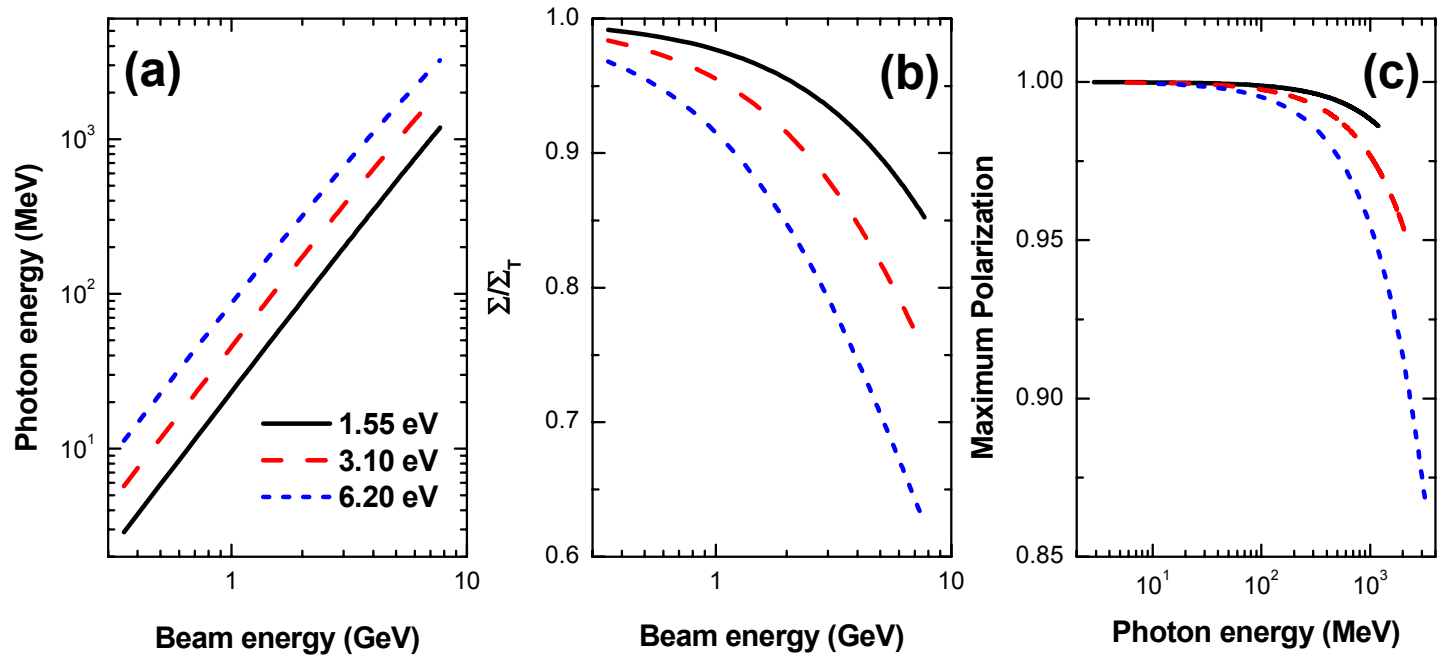

Fig. 1 (a) Gamma-ray photon energy and (b) total scattering cross section $\Sigma$ normalized to the Thomson scattering cross section of $\Sigma_{\mathrm{T}}=8 \pi \mathrm{r}_{\mathrm{e}}{ }^{2} / 3=0.665 \times 10^{-24} \mathrm{~cm}^{-2}$ as a function of the beam energy for laser photon energies of $1.55,3.1$, and $6.2 \mathrm{eV}$; (c) the maximum transferable linear polarization as a function of the photon energy.

\subsubsection{The booster case}

\section{a. Immediate accessible performances}

Using the formula developed in Appendix B, the calculated photon fluxes and the beam lifetime as a function of the photon energy are shown in Figs. 2 and 3 for beams stored in the booster and the APS storage ring, respectively. Here the highest achieved charge of $5 \mathrm{nC}$ in one bunch is used while the normal operation charge is 2-3 $\mathrm{nC}$ currently. The intrabeam scattering effect (see Appendix B, section 2) is included. An off-the-shelf laser system with $2.5 \mathrm{~W}$ of average power at the fundamental wavelength of $800 \mathrm{~nm}$ (Coherent RegA9000, see Table C1) is used for its closely matched repetition rate. Conversion efficiencies of $50 \%$ and $15 \%$ to the second harmonic (SH) and the
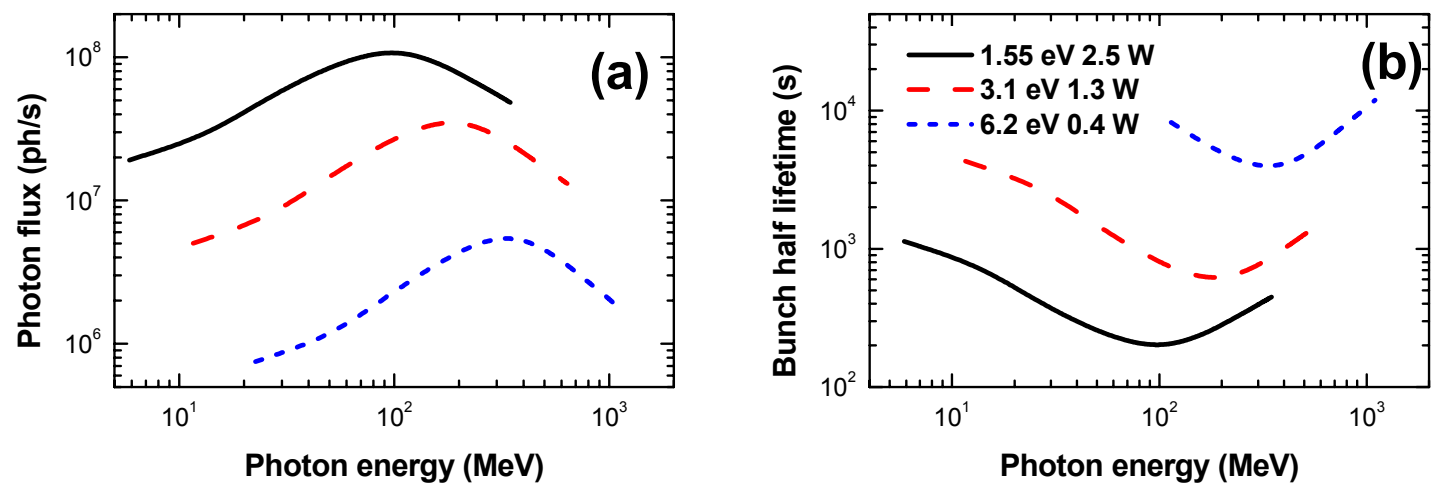

Fig. 2 (a) Photon flux and (b) beam half lifetime as functions of photon energy for the APS booster. Here a laser with 300-ps pulse duration, $2.5-\mathrm{W}$ average power at $800-\mathrm{nm}$ fundamental wavelength is used, and conversion efficiencies of $50 \%$ and $15 \%$ to the second harmonic $(\mathrm{SH})$ and the fourth harmonic $(\mathrm{FH})$ are assumed. The laser is assumed to have a repetition rate matching that of the booster beam rate of $815 \mathrm{kHz}$. A 5-nC charge is used in the calculation. Note that the bunch lifetime is for a single bunch. 
fourth harmonic $(\mathrm{FH})$, respectively, are used in the calculation. The photon fluxes depicted in Fig. 2 represent the immediately achievable performance of the system.

Clearly, a wide tuning range is easily achievable with the booster. Using the laser fundamental, the energy ranges from $6 \mathrm{MeV}$ to $100 \mathrm{MeV}$, while with the fourth harmonic of the same laser, the highest photon energy is 1 $\mathrm{GeV}$. The photon flux is about $10^{8} / \mathrm{s}$ at $100 \mathrm{MeV}$, while at $1 \mathrm{GeV}$ it drops to $2 \times 10^{6} / \mathrm{s}$.

\section{b. Possible upgrades}

To achieve higher photon fluxes, there are three upgrades to consider.

The first upgrade option is to push for the 10-nC booster stored charge as limited by the safety envelope. One way to accomplish this is to improve the rf cavity tuner so it is capable of compensating the effect of the large beam loading. The other way is to implement accumulation at low beam energies. A factor of two increase in the flux can be achieved this way.

The second possible upgrade is to improve beam quality. This essentially means replacing the existing magnets. Note that, by replacing the magnets, it is also possible to raise the storable beam energy to $7 \mathrm{GeV}$. Higher reptition rates are also possible. Together with the replaced power supplies for the magnets, at the time of this writing, the emittance has been improved from $130 \mathrm{~nm}$ rad to $90 \mathrm{~nm}$ rad by implementing a new lattice; a $30 \%$ improvement [8].

Finally, a more powerful laser can also help to improve the photon flux with a modest development effort. In fact, a 30-W, 75-MHz laser is under construction at the Jefferson National Accelerator Facility (JLab) [10].

Taking into account all of these possible improvements, especially the laser, a tenfold improvement in photon flux across the energy spectrum can be expected, which gives photon fluxes higher than $10^{9} / \mathrm{s}$ at $100 \mathrm{MeV}$ and $2 \times 10^{7} / \mathrm{s}$ at $1 \mathrm{GeV}$. Ultimately, the photon flux is limited by the duty cycle of the booster $(2 \mathrm{~Hz})$ and the maximum safety envelope.

\section{c. Disadvantages with the booster}

There are two obvious disadvantages with this booster scheme. The first one is that it relies on the relatively infrequent injection to the storage ring. Even in the current operation mode, 20 seconds out of the 2-min interval is needed to prepare the booster for injection. If in the future a more frequent injection is mandated by the storage ring operation, which is always a higher priority, the usable time for the envisioned $\gamma$-ray source will be further reduced.

The other disadvantage is directly related to the current beam quality and tagging. Clearly, the beam divergence in most of the cases is larger than $1 / \gamma$. This excludes the possibility of obtaining monoenergetic photons with a few percent by using collimating technique [3] without significantly improving the beam quality. At the same time, tagging is almost impossible due to the fact that the maximum number of bunches that can be put in the booster next to each other is 12 , separated only by $2.8 \mathrm{~ns}$. Even though the laser can be arranged to accommodate this beam pattern, the maximum flux allowed by a tagging system will be $10^{7} / \mathrm{s}$. Without significant hardware upgrades, it is impossible to store multiple bunches with even distribution around the booster.

\subsubsection{The APS storage ring case}

\section{a. Immediate accessible performances}

In comparison, the APS SR case seems to be more attractive, mainly due to the much higher beam quality and higher charge per bunch, multiple bunch capability, and most of all to the 'top-up' operation mode.

There are two possible locations for the Compton scattering setup as listed in Table 1: the insertion device (ID) position and the bending magnet (BM) position. We will only consider the BM location as the ID locations are all occupied.

Figures $3(\mathrm{a}-\mathrm{c})$ give the photon fluxes as a function of the stored bunch number using the current beam parameters in Table 1 for 800-, 400-, and 200-nm laser photons using Eq. (B5). A total store of $2.3 \times 10^{11}$ electrons is used. For tagging purposes, the photon production for each electron bunch is also given as the upper $\mathrm{x}$-axis. The laser used is the 3.5-W Spectra Physics Tsunami laser at $800 \mathrm{~nm}$. Again, conversion efficiencies of $50 \%$ and $15 \%$ to 


\section{Photons per scattering}
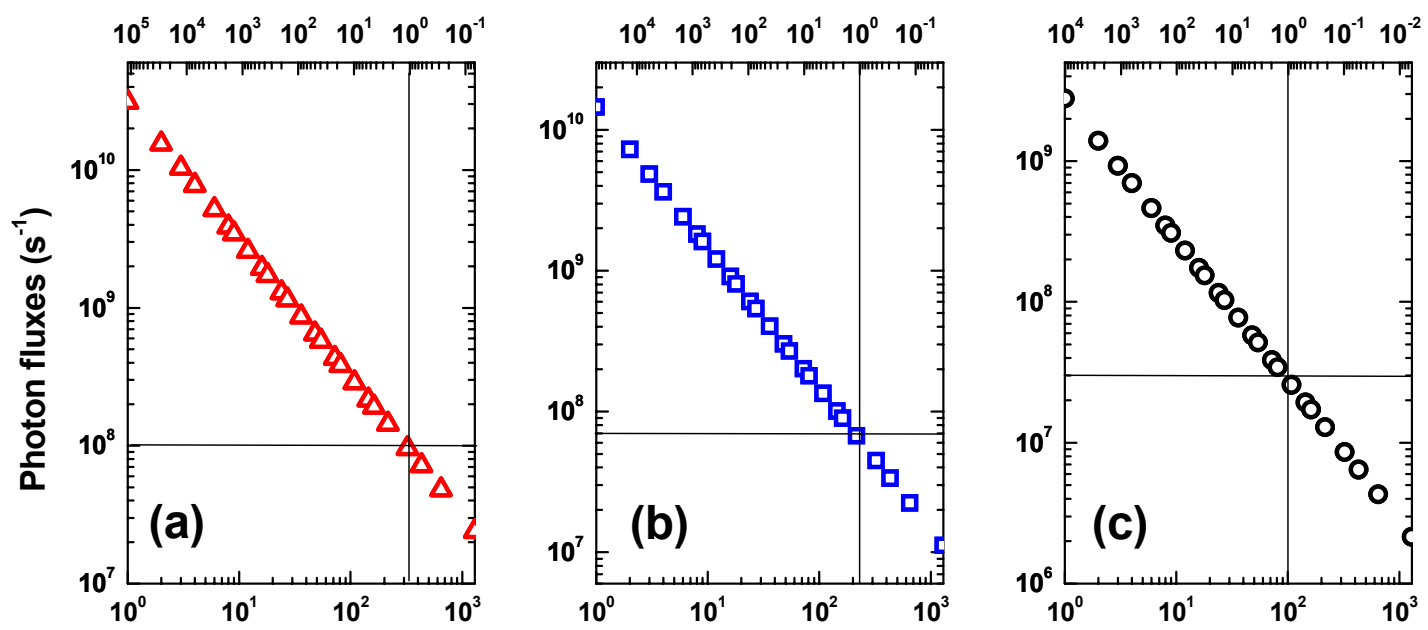

Number of bunches

Fig. 3 Photon fluxes as a function of the number of stored bunches for the APS SR with a 3.5-W laser working at the fundamental, $\mathrm{SH}$, and $\mathrm{FH}$ of $800 \mathrm{~nm}$ (a), $400 \mathrm{~nm}$ (b), and $200 \mathrm{~nm}$ (c). Conversion efficiencies to the SH and $\mathrm{FH}$ of $50 \%$ and $15 \%$ are used. The fundamental, $\mathrm{SH}$, and $\mathrm{FH}$ generate $\gamma$-ray photon energies of $1,1.7$, and $2.8 \mathrm{GeV}$, respectively.

the SH and the FH are assumed. The repetition rate of the laser is adjusted using an external cavity fitted with a cavity dumper [11] to match to the bunch repetition rate (see section 2.4).

Obviously, in Fig. 3, with the example laser and the beam parameters, it is not difficult at all to achieve photon fluxes higher than $10^{8}$ photons/s. For example, with the standard 24-bunch pattern, fluxes of $10^{9}, 6 \times 10^{8}$, and $10^{8}$ photons $/ \mathrm{s}$ at $1,1.7$, and $2.8 \mathrm{GeV}$ can be achieved, respectively. However, there are two limitations to be taken into account. The first one is the injection limit. Currently with 2-3 nC per injection every two minutes, the electron replenishing rate is $10^{8} \mathrm{e}^{-} / \mathrm{s}$. With the maximum achieved charge per injection of $4-5 \mathrm{nC}$ at the current interval of two minutes, the highest photon fluxes that can be supported are $1-2 \times 10^{8}$ photons/s.

The second limit is set by the requirement of tagging the electrons. Due to the high beam energy, the beam divergence is always bigger or equal to $1 / \gamma$, eliminating the possibility of using collimation for desired energy resolution [3]. Hence, to effectively identify the reaction events, one photon per scattering between the laser and the electron bunch is preferred. With this limitation in mind, using the current laser system, Fig. 3 shows that the photon fluxes achievable are $9.6 \times 10^{7}, 6.7 \times 10^{7}$, and $2.6 \times 10^{7} / \mathrm{s}$ at $1,1.7$, and $2.8 \mathrm{GeV}$, respectively. The repetition rates are 88,59 , and $29 \mathrm{MHz}$, with stored bunch number of 324, 216, and 108, respectively. These represent the immediately achievable performances with tagging and are generally one to two orders of magnitude better than the existing facilities at the same photon energy [3]. If the particular experiment does not need tagging, $10^{8} / \mathrm{s}$ is possible for all three energies.

\section{b. Possible upgrades}

Clearly, there are different aspects for the upgrades, i.e., for operations requiring tagging the electrons and for those that do not require tagging of the electrons.

For operation with no tagging requirement, only the flux is of interest. In this case, even with the current $3.5-\mathrm{W}$ laser, the maximum supported flux is $1-2 \times 10^{8} / \mathrm{s}$ if we double the charge per injection from the current $2-3 \mathrm{nC}$ to $4-5 \mathrm{nC}$, the highest ever demonstrated. To achieve the calculated fluxes of $1.3 \times 10^{9}$ and $6 \times 10^{8} / \mathrm{s}$ at $1 \mathrm{GeV}$ and 1.7 $\mathrm{GeV}$ with the standard 24 bunch pattern, more frequent injection is required. 
The current injection interval of two minutes is set to minimize the effect of transverse beam perturbation on the users of the synchrotron radiation. The perturbation arises from the kick to the stored beam with mismatched lattice to reduce the oscillation amplitude of the injected beam in order to accommodate the small-gap chambers in the beamline for efficient injection. The oscillation of the stored beam in turn induces the X-ray beam shift during the injection and an emittance growth shortly after it. The damping time of the induced betatron oscillation and beam size blowup is about $10 \mathrm{~ms}$. This injection scheme is named transverse injection.

Another injection scheme has been demonstrated and is under further study for operation; this is the longitudinal injection scheme [9]. In this injection scheme, a new lattice is required so that at the injection point, a large dispersion of the beam is generated, then the incoming beam is injected at an energy matching the dispersion. This way the incoming beam becomes a natural part of the stored beam except for a momentum difference. This momentun difference induces a synchrotron oscillation that damps with a time constant of $5 \mathrm{~ms}$. As no betatron oscillation is induced (on the stored beam), the X-ray beam is not affected as much.

Once implemented, this will significantly raise the photon flux at $1 \mathrm{GeV}$ and $1.7 \mathrm{GeV}$, up to $10^{9}$ and $6 \times 10^{8}$ photons/s, respectively, with the same 3.5-W laser and with 24-bunch pattern. Higher fluxes are also possible with less bunches or with higher-powered lasers.

For the operation where tagging is needed, from Fig. 1, we are clearly more limited by the laser power and the bunch repetition rate. To achieve this, 1296 bunches will be stored in the storage ring with a matching laser repetition rate. For operation at $10^{8} / \mathrm{s}$, the practical bunch repetition rate is $88 \mathrm{MHz}(352 \mathrm{Mz} / 4)$ or $117 \mathrm{MHz}(352$ $\mathrm{MHz} / 3$ ). From the calculation in Fig. 3, the flux at $1 \mathrm{GeV}$ is at about $6 \times 10^{8} / \mathrm{s}$ at $88 \mathrm{MHz}$ with the $3.5-\mathrm{W}$ laser in the calculation, while $2 \times$ and $7 \times$ more powerful lasers are needed to obtain the same performance at 1.7 and $2.8 \mathrm{GeV}$. The highest fluxes practicable with tagging capability correspond with the rf frequency of $352 \mathrm{MHz}$ in our case, which means a flux of $3.5 \times 10^{8}$ photons $/ \mathrm{s}$.

Fortunately, such a laser, though not commercially available, can be built with a modest effort, such as the one under development at JLab. The ultimate limitation is the radiation safety envelope of $10 \mathrm{nC}$ per injection and the duty cycle of $2 \mathrm{~Hz}$ of the booster, which translates to $10^{11}$ photons/s.

\subsection{Divergence, spectrum, and polarization}

The divergence of the photon beam is determined by the energy of the beam (i.e., $1 / \gamma$ ) and the divergence of the electron beam itself. From Eq. (A1) in Appendix A, the spectrum with a collimating angle $\Delta \theta$ is a convolution of beam (laser and electron) divergence and energy spread. In our cases, due to the high photon energy, electron tagging and photon collimation are needed for the desired energy resolution [3].

The polarization is proportional to that of the laser in both the circular and linear polarization cases. For the linear polarization case, the maximum transferable polarization will be reduced due to the spin flip in the electron bunches [3], as given in Eq. (A4) in Appendix A and shown in Fig. 1 (c).

\subsection{The laser}

In practice, electron tagging prefers higher duty cycle to higher peak brightness. In addition, the laser has to be synchronized to the electron beam with a jitter of several picoseconds for stable operation. This requires the laser system to be an oscillator-amplifier configuration or just an oscillator, which is temporally locked to the rf of the accelerators. A few of the off-the-shelf laser systems are listed in Table C1 in Appendix C for reference. The challenge is to tailor the laser to the required repetition rate while maintaining the power. We propose to use an external Fabry-Perot cavity with an acoustic optical modulator cavity dumper for this purpose [11]. In this case, the repetition rate can be tailored to any of the subharmonics of the fundamental rate.

In the above calculation, we used two commercial Ti:Sa laser systems with a few watts of output as examples. Higher laser power up to a few tens of watts can be obtained. For example, as mentioned earlier, a Ti:Sa laser of 30-W average power at $75 \mathrm{MHz}$ is under construction at JLab [10], and the development effort is described as modest. Another alternative, though more difficult to implement without modifying the electron beam trajectory, is to use an intracavity scattering scheme in an oscillator or with an external Fabry-Perot cavity. The intracavity optical power can be orders of magnitude higher than the direct output power from the laser. 
Table 2. Comparison of the Performance of the Proposed APS $\gamma$-Ray Source with Existing Sources

\begin{tabular}{|c|c|c|c|c|c|c|}
\hline & \multicolumn{2}{|l|}{ APS SR } & APS booster & $\begin{array}{l}\text { ESRF } \\
\text { GRAAL [3] }\end{array}$ & $\begin{array}{l}\text { SPring-8 } \\
\text { LEPS [3] }\end{array}$ & HIGS [3] \\
\hline Beam energy $(\mathrm{GeV})$ & \multicolumn{2}{|l|}{7} & $0.4-4$ & 6 & 8 & $0.2-1.3$ \\
\hline$\gamma$-ray energy $(\mathrm{GeV})$ & \multicolumn{2}{|l|}{$1,1.7,2.8$} & $0.005-1.0$ & $0.55-1.50$ & $1.5-2.4$ & $<0.220$ \\
\hline $\begin{array}{l}\text { Flux (photons/s) } \\
\text { Immediate } \\
\text { Foreseeable upgrade } \\
\text { Machine limit }\end{array}$ & $\begin{array}{l}\text { Tag } \\
3 \times 10^{7}-10^{8} \\
10^{8} \\
3.5 \times 10^{8}\end{array}$ & $\begin{array}{l}N \text { tag } \\
1-2 \times 10^{8} \\
3 \times 10^{8}-3 \times 10^{9} \\
10^{11}\end{array}$ & $\begin{array}{l}\mathrm{N} \text { tag } \\
2 \times 10^{6}-10^{8} \\
2 \times 10^{7}-10^{9} \\
10^{11}\end{array}$ & $3 \times 10^{6}$ & $5 \times 10^{6}$ & $10^{6}-10^{10}$ \\
\hline
\end{tabular}

We use a Rayleigh length of 300 ps for the fundamental in our calculation. Using Eq. (B6) in Appendix B, the optimum laser spot size is $\sigma_{0}=76 \mu \mathrm{m}, 38$, and $19 \mu \mathrm{m}$ for the fundamental, second, and fourth harmonics, respectively, for a common set of focusing optics. We further assume $50 \%$ and $15 \%$ conversion efficiencies for the second and fourth harmonics.

\subsection{Comparison with other $\gamma$-ray sources}

Table 2 summarizes the immediate and future performances of the proposed APS storage ring/booster $\gamma$-ray sources and compares those with the performance of GRAAL [4], LEPS [5], and future HIGS [7]. It is believed that HIGS will be the brightest $\gamma$-ray source when commissioned.

In Table 2 there are three flux entries for the APS sources, and each entry is divided into two columns, with and without tagging. The entry 'immediate' represents those that can be available now with existing commercial laser systems and no change in the hardware and operation mode for the accelerator systems. The entry 'foreseeable' represents those with achievable laser performances after modest development effort and changes in the operation modes of the accelerator systems. The entry 'machine limit' represents those that can be achieved only after full accelerator system hardware upgrades and operation scheme optimization, together with state-of-the-art laser system upgrades.

To summarize from Table 2, the major advantages of the two proposed APS $\gamma$-ray sources are:

1) High photon fluxes of up to $10^{8} / \mathrm{s}$, about one to two orders of magnitude higher than those at GRAAL and LEPS;

2) An ultrabroad tuning range from $5 \mathrm{MeV}$ to $1 \mathrm{GeV}$ for the booster source and high photon energy of 2.8 $\mathrm{GeV}$ for the storage ring in comparison with the 2- to $200-\mathrm{MeV}$ range at $\mathrm{HIGS}$, and the $1.5 \mathrm{GeV}$ and $2.4 \mathrm{GeV}$ available at GRAAL and LEPS, respectively;

3) With developing laser technologies and upgrades to the operation mode of the APS machines, photon fluxes of $10^{11} / \mathrm{s}$ are possible, limited only by the machines' safety envelopes.

\section{Summary}

Due to the particular operation mode of the APS storage ring and booster, our calculations show that with currently available laser technology and the existing operation lattice, it is possible to build $\gamma$-ray radiation sources with high photon fluxes at photon energies from a few $\mathrm{MeV}$ to $2.8 \mathrm{GeV}$. If built, either one will become an extremely useful $\gamma$ ray source for nuclear physics applications.

\section{Acknowledgement}

Many people have contributed to this feasibility study, including B. Berman and J. Feldman at George Washington University. G. Neil at JLab and V. Litvinenko at HIGS also contributed very useful comments and criticisms. This work is supported by the U. S. Department of Energy, Office of Basic Energy Sciences, under Contract No. W-31109-ENG-38. 


\section{References}

1. R. H. Milburn, Phys. Rev. Lett. 10, 75 (1963).

2. F. R. Arutyunyan, V. A. Tumanian, Phys. Lett. 4, 176 (1963).

3. A. D'Angelo et al., NIM A455, 1 (2000), and references therein.

4. J.P. Bocquet et al., Nucl. Phys. A 622, 124c (1997).

5. T. Nakano et al., Nucl. Phys. A 684, 71c (2001).

6. A. M. Sandorfi et al., IEEE Trans. NS-30, B 3083 (1983).

7. T. S. Carman, NIM A 378, 1 (1996); HIGS collaboration, "A Proposal for the Support of a Free -Electron Laser Generated High-Intensity $\gamma$-Ray Source for Nuclear Physics," available on-line at http://www.tunl.duke.edu/Local/proposal/higs/.

8. This will result in an approximately $30 \%$ improvement of the photon flux over those in this note for the booster, especially for high-energy operations where the intrabeam scattering effect is negligible.

9. Y. Chae, private communication.

10. G. Neil, private communication.

11. R. J. Jones and J. Ye, Opt. Lett. 27, 1848 (2002).

12. V. B. Berestetskii, E. M. Lifshitz, and L. P. Pitaevskii, Quantum Electrodynamics, $2^{\text {nd }}$ edition, Chapter X (Pergamon, New York, 1994). 


\section{Appendix A: Basics of Compton scattering}

\section{A.1 $\gamma$-ray photon energy and scattering cross section}

For head-on scattering $(\phi=\pi)$, the energy of the scattered photons scales as

$$
E_{s}=\frac{4 \gamma^{2} E_{L}}{1+\frac{4 \gamma E_{L}}{m c^{2}}+(\theta \gamma)^{2}} .
$$

Here $\gamma$ is the relativistic factor of the electron beam, $E_{\mathrm{L}}$ is the laser photon energy, $m$ is the electron mass, and $\theta$ is the scattering angle of the photon from the electron propagation direction. The total scattering cross section is [12]

$$
\Sigma=\frac{2 \pi r_{e}^{2}}{x}\left[\left(1-\frac{4}{x}-\frac{8}{x^{2}}\right) \ln (1+x)+\frac{1}{2}+\frac{8}{x}-\frac{1}{2\left(1+x^{2}\right)}\right] .
$$

Here $r_{\mathrm{e}}$ is the classical electron radius, and

$$
x=\frac{2 \gamma E_{L}(1-\beta \cos \phi)}{m c^{2}}=\frac{4 \gamma E_{L}}{m c^{2}} .
$$

\section{A.2 Spectrum}

The full spectrum of the $\gamma$-ray is a convolution of the energy spread and divergence of the laser beam and the electron bunch.

\section{A.3 Polarization}

The degree of polarization of the scattered photons is proportional to that of the laser beam for both linear and circular polarization, and the polarization is highest at the Compton edge (highest photon energy) where the spin-flip amplitude for highly relativistic electrons vanishes. In the linear polarization case, there is a drop of the maximum transferable polarization at high photon energy [3]:

$$
P_{\gamma \max }=P_{\text {Laser }} \frac{2}{2+\frac{(1-a)^{2}}{a}} \text {. }
$$

Here $a=1 /(1+x)$, with $x$ defined in Eq. (A3).

\section{Appendix B: Photon flux and electron beam lifetime}

\section{B.1 Calculation of photon flux}

Consider an electron bunch with the following distribution

$$
f_{e}=\frac{N_{b}}{(2 \pi)^{3 / 2} \sigma_{x} \sigma_{y} \sigma_{z}} \exp \left(-\frac{x^{2}}{2 \sigma_{x}^{2}}-\frac{y^{2}}{2 \sigma_{y}^{2}}-\frac{(z-c t)^{2}}{2 \sigma_{z}^{2}}\right),
$$

with transverse rms beam size of

$$
\begin{aligned}
& \sigma_{x}=\sqrt{\frac{\varepsilon_{0} \beta_{x}+\eta_{x}^{2} \sigma_{\delta}^{2}}{1+k}} \frac{\gamma}{\gamma_{0}}, \\
& \sigma_{y}=\sqrt{\frac{k \varepsilon_{0} \beta_{y}}{1+k} \frac{\gamma}{\gamma_{0}}}
\end{aligned}
$$


and an rms longitudinal bunch size of $\sigma_{\mathrm{z}}$. Here $N_{\mathrm{b}}$ is the total number of electrons in one bunch, $N_{\mathrm{b}}=N_{\mathrm{e}} / n_{\mathrm{b}}$; here $N_{\mathrm{e}}$ is the total number of electron stored in the ring and $n_{\mathrm{b}}$ is the total number of evenly distributed bunches. For the APS storage ring, the maximum bunch number is $1296 . \varepsilon_{0}$ is the emittance at beam energy $\gamma_{0}, k$ is the coupling factor, and $\beta_{x, y}$ are the beta functions of the electron beam, the equivalent of the Rayleigh range in optics. $\sigma_{\delta}$ is the rms relative momentum spread and $\eta_{\mathrm{x}}$ is the dispersion function, which has a negligible effect here.

Similarly, we assume the laser has the following distribution at the focus:

$$
f_{p}=\frac{N_{p}}{(2 \pi)^{3 / 2} \sigma_{0}^{2} \sigma_{t}} \exp \left(-\frac{x^{2}+y^{2}}{2 \sigma_{0}^{2}}-\frac{(z / c+t)^{2}}{2 \sigma_{t}^{2}}\right),
$$

with an rms pulse width of $\sigma_{t}$ and beam waist $w_{0}=2 \sigma_{0}$. Here $N_{\mathrm{p}}$ is the total number of photons per pulse, $N_{\mathrm{p}}=F_{\mathrm{p}} / f_{\mathrm{b}}$, with the bunch repetition rate $f_{\mathrm{b}}=n_{\mathrm{b}} f$, where $f$ is the revolution frequency of the storage ring. The total number of $\gamma$ ray photons per shot is calculated as

$$
N_{\gamma}=\Sigma \iiint_{x, y, z, t} f_{e} f_{p} d x d y d z d t
$$

The integration yields

$$
N_{\gamma}=\Sigma \frac{N_{b} N_{p}}{2 \pi \sqrt{\sigma_{0}^{2}+\sigma_{x}^{2}} \sqrt{\sigma_{0}^{2}+\sigma_{y}^{2}}}=\Gamma \frac{N_{e} F_{p}}{n_{b}^{2} f},
$$

where

$$
\Gamma=\frac{\Sigma}{2 \pi \sqrt{\sigma_{0}^{2}+\sigma_{x}^{2}} \sqrt{\sigma_{0}^{2}+\sigma_{y}^{2}}} .
$$

Obviously, with given $\sigma_{\mathrm{x}}$ and $\sigma_{\mathrm{y}}, \sigma_{0}$ is the key factor that determines the flux: the smaller the $\sigma_{0}$, the higher the flux. On the other hand, Eqs. (B4-B5) require the laser Rayleigh length, $Z_{\mathrm{R}}=\pi w_{0}{ }^{2} / \lambda$, to be equal to or larger than the larger of the FWHM of the laser pulse $\mathrm{c} \tau_{\mathrm{L}}$ and the electron bunch length $2 \sqrt{2 \ln 2} \sigma_{z}$ (the beta functions of the electron beams are normally very large, hence are not considered a limitation). Assuming that the laser pulse duration is the larger, the optimum laser spot size is approximately
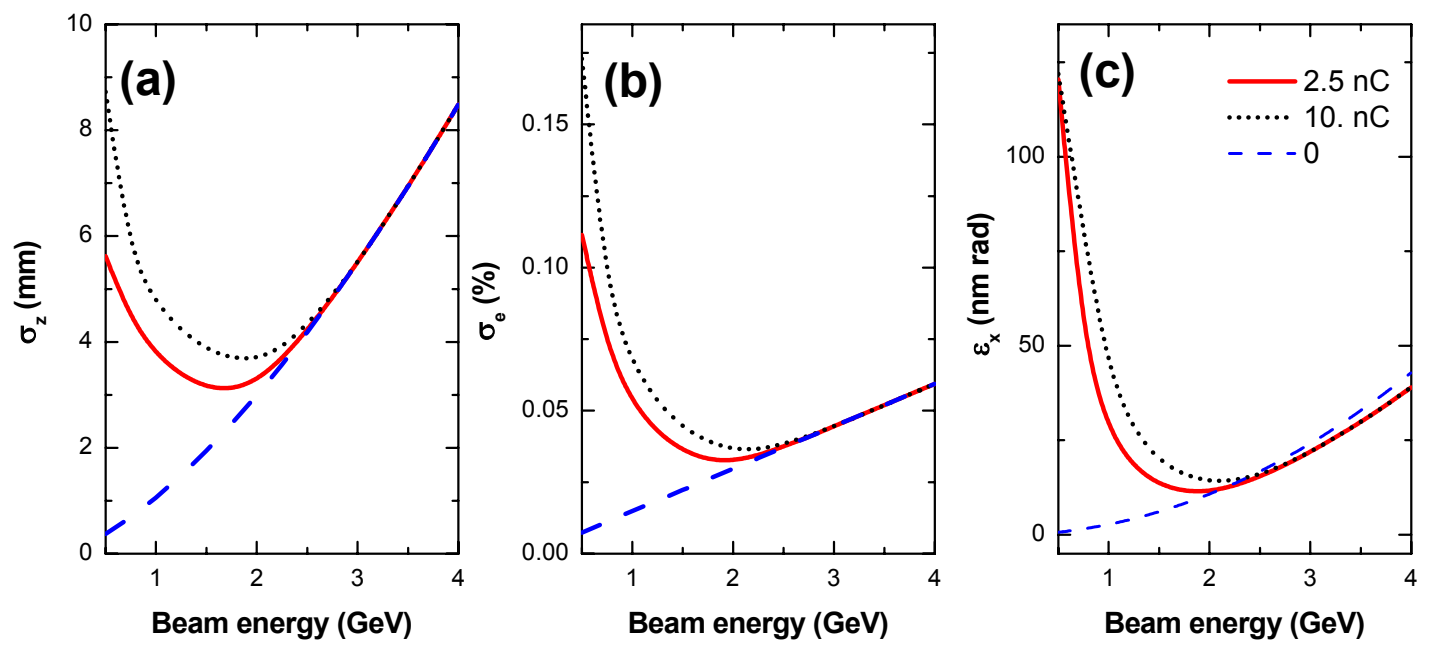

Fig. B1 The effect of intrabeam scattering on the electron (a) bunch length, (b) energy spread, and (c) emittance, for ideal (0$\mathrm{nC}), 2.5-\mathrm{nC}$, and 10-nC charges for the APS booster. 
Table C1. Performance of the Off-the-Shelf Ti:Sa Lasers

\begin{tabular}{|l|l|l|l|}
\hline Make and model & Energy per pulse $^{a}$ & Rep rate & Average power \\
\hline $\begin{array}{l}\text { Coherent } \\
\text { RegA9000 }\end{array}$ & $\begin{array}{l}5 \mu \mathrm{J} \text { compressed } \\
10 \mu \mathrm{J} \text { uncompressed }\end{array}$ & $250 \mathrm{kHz}$ & $2.5 \mathrm{~W}$ \\
\hline Quantronix & $\begin{array}{l}>5 \mathrm{~mJ} \text { compressed } \\
10 \mathrm{~mJ} \text { uncompressed }\end{array}$ & $1 \mathrm{kHz}$ & $10 \mathrm{~W}$ \\
\hline $\begin{array}{l}\text { Spectra Physics } \\
\text { Tsunami }\end{array}$ & $40 \mathrm{~nJ}$ & $80 \mathrm{MHz}$ & $3.5 \mathrm{~W}$ \\
\hline $\begin{array}{l}\text { Coherent } \\
\text { Mira }\end{array}$ & $20 \mathrm{~nJ}$ & $80 \mathrm{MHz}$ & $1.4 \mathrm{~W}$ \\
\hline
\end{tabular}

a. In deriving the uncompressed pulse energy, the compressor efficiency is assumed to be $50 \%$.

$$
\sigma_{0} \approx \sqrt{\frac{c \tau_{L} \lambda}{4 \pi}} .
$$

When using lasers at different wavelengths, the beam waist scales with the wavelength if the focusing optics is maintained at the same $f$-number.

\section{B.2 Photon flux and electron bunch lifetime}

Rewriting Eq. (B5) as a production rate per shot $r=N_{\gamma} / N_{\mathrm{b}}=\Gamma N_{\mathrm{p}}$, for a repetition rate of $f$ for each bunch and $f r<<1$, the total flux $F$ and the beam half lifetime $T$ are

$$
\begin{aligned}
& F=r n_{b} N_{b} \sum_{j=0}^{f-1}(1-r)^{j}=N_{e}\left[1-(1-r)^{f}\right] \approx r f N_{e}=\Gamma \frac{F_{p} N_{e}}{n_{b}}, \\
& T=-\frac{1}{f} \frac{\ln 2}{\ln (1-r)} \approx \frac{\ln 2}{f r}=\frac{n_{b} \ln 2}{\Gamma F_{p}} .
\end{aligned}
$$

Note that, in case where tagging is needed, hence $N_{\gamma} \approx 1$, we have $F \approx f_{\mathrm{b}}$, the bunch repetition rate. Hence the highest flux achievable is limited by the rf of the accelerators systems. Equation (B7) does not include the effect of natural decay of the beam.

\section{B.3 Effect of the intrabeam scattering}

In a storage ring, the beam quality is determined by quantum excitation and radiation damping of the electrons. At low beam energies, the intrabeam scattering will cause the bunch length, energy spread, and emittance to grow. These effects are shown in Fig. B1 (a-c), respectively, for the APS booster at bunch charges of 10, 2.5, and $0 \mathrm{nC}$.

\section{Appendix C: Off-the-shelf laser systems}

The performances of several off-the-shelf laser systems are listed in Table $\mathrm{C} 1$ for reference. Note that these commercial lasers only represent the standard performance one can achieve with such systems. With modest development effort, laser systems with performances far exceeding these can be built [11]. 